\title{
Modelado del Proceso Convencional de Colada Continua de Aceros Libres de Interticios
}

Bruno A. Pereira, José A. de Castro, Alexandre J. da Silva y Jorge A. R. Durán

Universidade Federal Fluminense, Escola de Engenharia Industrial Metalúrgica de Volta Redonda, Av. dos Trabalhadores, ${ }^{\circ}$ 420, CEP: 27255-125, Volta Redonda RJ - Brasil.

(e-mail: brunoap@metal.eeimvr.uff.br)

Recibido Dic. 28, 2009; Aceptado Feb. 15, 2010; Versión Final recibida Abr. 19, 2010

\begin{abstract}
Resumen
El objetivo del trabajo fue desarrollar un modelo matemático capaz de simular el proceso convencional de colada continua de aceros IF (Interstitial Free), y determinar las condiciones operacionales óptimas. La formulación del modelo incluye las ecuaciones de momento lineal de materiales líquidos y solidificados junto con la evolución térmica de la plancha. Las ecuaciones diferenciales y las condiciones de contorno son resueltas numéricamente mediante volúmenes finitos. Ciertas condiciones de contorno como el flujo de calor para el enfriamiento en cada región, además de la resistencia al flujo de la capa de fundente y las oscilaciones en la región del molde, son especificadas. Las predicciones del modelo fueron comparadas con datos industriales para las condiciones de las coladas continuas convencionales y extendidas a aceros IF.
\end{abstract}

Palabras clave: colada continua, acero IF, modelado matemático, simulación computacional, volúmenes finitos.

\section{Modeling of the conventional process of continuous Interstitial Free casting}

\begin{abstract}
The purpose of this work was to develop a mathematical model able to simulate the continuous casting process of IF steel (Interstitial Free).and to determine the optimum operating conditions. The model formulation involves the momentum equations of liquid and solidified materials coupled with the temperature evolution of the slab. The differential equations and boundary conditions are numerically solved using the finite volume technique with appropriated boundary conditions for each region of the casting machine. The model predictions were compared with industrial data for conventional continuous casting conditions and extended to IF steel.
\end{abstract}

Keywords: continuous casting, IF steel, mathematical model, computational simulation, finite volumes. 


\section{INTRODUCIÓN}

El desarrollo en el procesamiento de datos permite considerables avances tecnológicos en varios sectores metalúrgicos. El progreso en simulación computacional permite un mejor conocimiento del proceso de colada continua y ha permitido realizar algunas investigaciones sobre el efecto de los parámetros del proceso en la seguridad de las operaciones. La máquina de colada Fig. 1, permite la solidificación continua de metal líquido alimentado por un distribuidor a través de una válvula sumergida. Un intenso flujo de calor es impuesto en el molde y la capa sólida que se forma es sacada para afuera. Mientras tanto, agua de enfriamiento es aplicada hasta que la plancha es cortada y descargada en una mesa rodante.

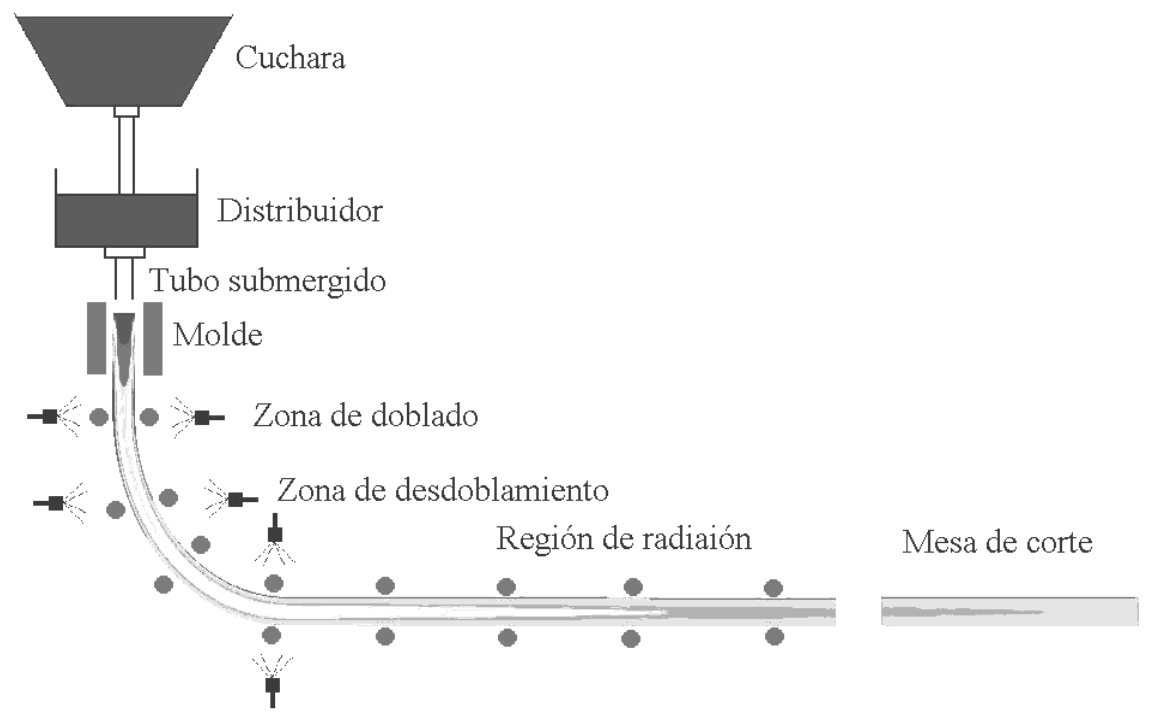

Fig. 1 - Esquema del proceso de colada continua.

Debido a la complejidad del proceso que mezcla transmisión de calor con transformaciones de fase, la predicción de parámetros tecnológicos y la optimización del proceso normalmente se hacen mediante correlaciones empíricas. No obstante, con el desarrollo de eficientes métodos numéricos y computadoras, la tarea de modelamiento se ha hecho posible y ha contribuido para el aumento del entendimiento del problema, lo que ha favorecido la creación de nuevas técnicas operacionales. Hoy en día es posible investigar la producción de varios tipos de aceros con bajo costo y alta eficiencia de material. Varios trabajos se han focalizado en el estudio del comportamiento del material dentro del molde de la máquina de colada continua debido a su importancia en la productividad y en la calidad final da las planchas producidas. El molde oscilante es un componente importante de la máquina y tiene una fuerte influencia en los defectos superficiales y en la distribución de temperatura dentro del molde (Lan y Khodadadi, 2001; Ha et al., 2003; Peng et al., 2005). El análisis de transmisión de calor durante la solidificación se ha realizado tradicionalmente por métodos analíticos y numéricos. Mientras que los métodos analíticos son más elegantes, estos requieren una serie de consideraciones que generalmente originan simplificaciones considerables lo que puede llevar a resultados irreales. Por estas razones los métodos numéricos se utilizan ampliamente. Entre estos los más comunes están: diferencias finitas (Choudhary et al., 1993; Shi y Guo, 2004), elementos finitos (Thomas et al., 1990; Janik et al., 2004), volúmenes finitos (Huespe et al., 2000) y elementos de contorno (Fic, 2000). Estos métodos son capaces de formular y resolver las ecuaciones de transmisión de calor. En este trabajo se utiliza un modelamiento matemático tridimensional para simular el proceso de colada continua de planchas de acero e investigar las condiciones de fundición necesarias para producir planchas de acero IF (Interstitial Free) convencionales. Se calcula la distribución de temperaturas utilizando la ecuación de transmisión de calor bajo la consideración de que las condiciones son estacionarias y numéricamente resolubles, utilizando el método de los volúmenes finitos. El modelo matemático se utiliza entonces para predecir el campo de temperaturas en la chapa de acero, desde el alimentador hasta la mesa de corte. El modelo usa informaciones del proceso para definir condiciones de enfriamiento en las fronteras y velocidades de fundición reales. 


\section{MATERIALES Y MÉTODOS}

\section{Modelamiento matemático}

El modelo se basa en las ecuaciones de transporte de momento lineal y de energía acopladas con la velocidad de solidificación. El dominio se restringe a la vena de colada continua desde la entrada de metal caliente a través de la válvula sumergida hasta la mesa de salida donde se corta la plancha. El movimiento del metal líquido y las regiones solidificadas se modelan como fluidos no newtonianos con una viscosidad aparente seleccionada. Las ecuaciones 1 y 3 representan la descripción del modelo. Una ecuación adicional (Ec. 7) se utiliza para determinar la fracción solidificada.

Las condiciones de frontera para las ecuaciones del momento lineal son consideradas en la entrada y salida de la plancha mientras que las regiones del molde y de los rodillos son consideradas como de deslizamiento perfecto. Para las condiciones de frontera relacionadas con la transmisión de calor la vena fue dividida en varias regiones y las velocidades de enfriamiento fueron especificadas mediante un coeficiente efectivo de transmisión del calor el que, por su vez, es una función del flujo de agua y de la temperatura. En la región final se incluyó también la condición de radiación.

Las simulaciones numéricas se llevaron a cabo dividiendo el dominio computacional en 8 sectores: el molde, el pie del rodillo, cuatro zonas de enfriamiento secundario y la mesa de corte. La longitud de cada zona se muestra en la tabla 1.

Tabla 1: Dimensiones de las zonas de enfriamiento

\begin{tabular}{|l|l|}
\hline Molde & $0,9 \mathrm{~m}$ \\
\hline Pie del Rodillo & $0,422 \mathrm{~m}$ \\
\hline Zona de Doblado & $2,416 \mathrm{~m}$ \\
\hline Región 1 & $1,257 \mathrm{~m}$ \\
\hline Región 2 & $3,828 \mathrm{~m}$ \\
\hline Región 3 & $3,828 \mathrm{~m}$ \\
\hline Región 4 & $4,492 \mathrm{~m}$ \\
\hline Mesa de corte & $9,315 \mathrm{~m}$ \\
\hline
\end{tabular}

La tarea de modelar consiste en resolver simultáneamente las ecuaciones del impulso, transmisión del calor y transformaciones de fase. La Ec. 1 representa el equilibrio del momento lineal:

$\rho \frac{\partial u_{i}}{\partial x_{j}}=-\frac{\partial p}{\partial x_{i}}+\frac{\partial}{\partial x_{j}}\left[\mu_{e f f}\left(\frac{\partial u_{i}}{\partial x_{j}}\right)\right]$

donde $u$ es el campo de velocidades, $p$ la presión y $\mu_{\text {eff }}$ es la viscosidad efectiva (Moreira y Castro, 2008), Ec. 2.

$\mu_{\text {eff }}=\frac{\bar{\sigma}}{3 \dot{\bar{\varepsilon}}}$

Donde $\bar{\sigma}$ es la tensión promedio en el material y $\dot{\bar{\varepsilon}}$ es la tasa de deformación efectiva presentada por (Zienkiewicz, 1978)

La distribución de temperatura en la plancha durante el proceso de colada continua puede ser descrita por la ecuación de conducción del calor tridimensional. Para el régimen estacionario se tiene: 


$$
\frac{\partial\left(\rho u_{j} c_{P} T\right)}{\partial x_{j}}=\left[\frac{\partial}{\partial x_{j}}\left(k \frac{\partial T}{\partial x_{j}}\right)\right]+S
$$

donde $T$ es la temperatura, $k$ la conductividad térmica, $c_{p}$ el calor específico, $\rho$ la densidad y $S$ el cambio de energía asociado con la solidificación y dado por la Ec. 6:

Para la fase sólida el calor específico y la conductividad térmica fueron asumidas como funciones de la temperatura, como mostrado en las Ec. $4-5$, respectivamente definidas por Holman (Holman, 1982) y Colin (Colin y Brandes, 1976).

$\mathrm{k}=\lambda-\gamma \times 10^{-2} \mathrm{~T}$

$c_{P}=a+b T+c T^{1 / 2}$

$S=\rho L \frac{\partial f_{S}}{\partial t}$

donde $\lambda, \mathrm{y}, \mathrm{a}, \mathrm{b}$ y c son constantes referentes a cada material, $L$ es el calor latente de fusión y $f_{s}$ la fracción sólida que puede calcularse de la siguiente forma:

$$
f_{S}=1-\left(\frac{T_{f}-T}{T_{f}-T_{l}}\right)
$$

donde $T_{f}$ es la temperatura de solidus y $T_{l}$ es la temperatura de líquidus extraída del diagrama de equilibrio.

\section{Condiciones de frontera}

La caracterización de las diferentes zonas fue hecha imponiendo las condiciones de frontera características en cada una de ellas. En el molde y en el pie del rodillo existe agua de enfriamiento en los cuatro lados (dos grandes, interno y externo, y dos más estrechos, izquierdo y derecho) mientras que en las otras zonas hay solo dos lados (interno y externo, ambos grandes).

El flujo de calor de la plancha para la superficie en las áreas enfriadas con agua se describe por la ecuación de Newton:

$-k \frac{\partial T}{\partial x_{j}}=h_{e f f}\left(T_{\text {sur }}-T_{e}\right)+\sigma \varepsilon\left(T_{\text {sur }}^{4}-T_{e}^{4}\right)$

donde $h$ es el coeficiente de transmisión de calor, $T_{\text {sur }}$ es la temperatura de la plancha y $T_{e}$ la temperatura ambiente, $\sigma$ es la constante de Stefan-Boltzman y $\varepsilon$ la emisividad.

El coeficiente de intercambio de calor en las zonas salpicadas (pie del rodillo, dobladora y zona de enfriamiento secundario) fue calculado a partir del equilibrio de entalpía del agua.

$h_{e f f}=\frac{m_{w} \times c_{p} \times \Delta T}{A\left(T_{\text {sur }}-T_{e}\right)}$

donde $m_{w}$ es el flujo de agua, $c_{p}$ es el calor específico del agua, A es el área interfacial y $\Delta T$ es la diferencia de temperatura del agua introducida para la puesta a punto del sistema de enfriamiento. 
En la región del molde se utilizó un coeficiente de intercambio de calor para el acero que depende del tiempo en que el mismo permanece en esa condición. Este coeficiente considera una resistencia térmica debido a la formación de una brecha por el aire (Silva, 1996).

$h_{\text {mold }}=1004,6 \cdot \exp \left(-0,02 t_{m}\right), \mathrm{W} / \mathrm{m}^{2} \mathrm{~K}$

donde $t_{m}$ es el tiempo de permanencia del acero en el molde que se calcula utilizando a velocidad de la plancha $V_{c}$ y la altura del molde $Y$.

$t_{m}=\frac{Y}{V_{c}}$

\section{Metodología Numérica}

Las ecuaciones para el movimiento y la transmisión del calor fueron discretizadas utilizando el método de los volúmenes finitos (MVF) en el sistema general de coordenadas recomendado por (Melaaen, 1992). La integración se realiza mediante control del volumen Fig. 3, Ec. 12. El producto final de esta operación es una ecuación algebraica resumida en la Ec.13 con coeficientes obtenidos de acuerdo con el llamado esquema de ley de potencia (Patankar, 1980).

$\int_{\delta t} \int_{\delta V} \frac{\partial(\rho \phi)}{\partial t} d v d t+\int_{\delta t} \int_{\delta V}\left[\operatorname{div}\left(\rho \vec{U} \phi-\Gamma_{\phi} \operatorname{grad}(\phi)\right)\right] d v d t=\int_{\delta t} \int_{\delta V} S_{\phi} d v d t$

donde $\phi$ representa la variable dependiente, $\vec{U}$ el flujo convective y $\Gamma$ el coeficiente de transporte.

$a_{P} \phi_{P}=a_{W} \phi_{W}+a_{E} \phi_{E}+a_{B} \phi_{B}+a_{T} \phi_{T}+a_{S} \phi_{S}+a_{N} \phi_{N}+b$

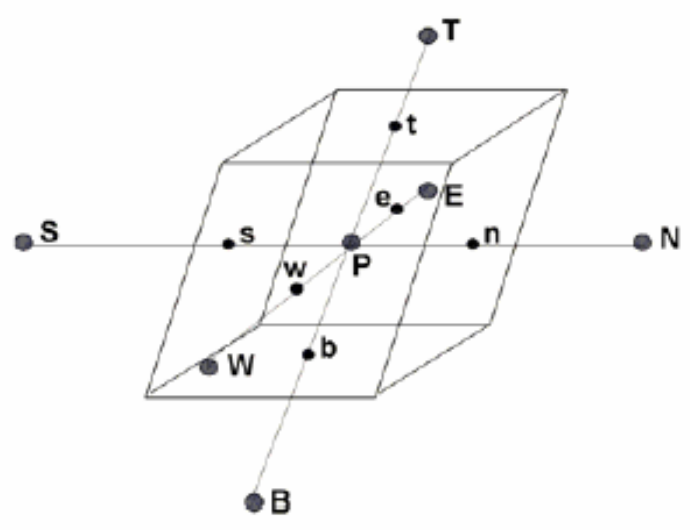

Fig. 2: Control del volumen

El movimiento del líquido y del metal solidificado fue obtenido utilizando el algoritmo SIMPLE, donde las componentes de las velocidades y de la presión se determinan interactivamente. El método de la entalpia fue utilizado para modelar el campo de temperatura asociado con el proceso de solidificación. La solución numérica de las ecuaciones algébricas resultantes del método de discretización demanda un gran trabajo computacional. El código usa el método de la línea por línea basado en la solución tridiagonal de matrices. El proceso interactivo ADI (Alternated Direct Implicit) se utiliza como un solucionador común de ecuaciones.

\section{RESULTADOS}

En las simulaciones de colada continua del acero llevadas a cabo en este trabajo, además de los datos de la tabla 1, fueron utilizados también los datos industriales de la tabla 2 y las condiciones de enfriamiento impuestas para cada sector de la máquina. 
Tabla 2 - Datos usados en las simulaciones realizadas en este estudio.

\begin{tabular}{|l|l|l|}
\hline \multicolumn{1}{|c|}{ Parámetros } & \multicolumn{1}{c|}{ Acero C-Mn } & \multicolumn{1}{c|}{ Acero IF } \\
\hline Ancho de la chapa & $1,6 \mathrm{~m}$ & $1,6 \mathrm{~m}$ \\
\hline Espesor de la chapa & $0,255 \mathrm{~m}$ & $0,255 \mathrm{~m}$ \\
\hline Temperatura de caída & $1.574{ }^{\circ} \mathrm{C}$ & $1.574{ }^{\circ} \mathrm{C}$ \\
\hline Velocidad de colada & $0,81 \mathrm{~m} / \mathrm{min}$ & $0,81 \mathrm{~m} / \mathrm{min}$ \\
\hline Temperatura del agua de enfriamiento & $30^{\circ} \mathrm{C}$ & $30^{\circ} \mathrm{C}$ \\
\hline Temperatura ambiente & $40^{\circ} \mathrm{C}$ & $40^{\circ} \mathrm{C}$ \\
\hline Temperatura de inicio de solidificación & $1539^{\circ} \mathrm{C}$ & $1533^{\circ} \mathrm{C}$ \\
\hline Temperatura de fin de solidificación & $1534^{\circ} \mathrm{C}$ & $1528^{\circ} \mathrm{C}$ \\
\hline Material de la chapa & Acero carbono $(0,15 \% \mathrm{C})$ & Acero IF $(0,003 \% \mathrm{C})$ \\
\hline Emisividad & 0,6 & 0,6 \\
\hline Conductividad térmica en la fase líquida & $41,0 \mathrm{~W} / \mathrm{m} \mathrm{K}$ & $12,0 \mathrm{~W} / \mathrm{m} \mathrm{K}$ \\
\hline Calor específico en la fase líquida & $749,5 \mathrm{~J} / \mathrm{kg} \mathrm{K}$ & $448,5 \mathrm{~J} / \mathrm{kg} \mathrm{K}$ \\
\hline Densidad & $7.830 \mathrm{~kg} / \mathrm{m}^{3}$ & $7.640 \mathrm{~kg} / \mathrm{m}^{3}$ \\
\hline Calor latente de solidificación & $2,07 \times 10^{8} \mathrm{~J} / \mathrm{m}^{3}$ & $2,07 \times 10^{8} \mathrm{~J} / \mathrm{m}^{3}$ \\
\hline
\end{tabular}

El acero IF fue escogido en este trabajo debido a que es ampliamente utilizado en diferentes sectores de industrias como la automovilística y la electro electrónica. Esta clase de acero presenta excelentes propiedades para estas industrias debido a la baja concentración de carbón e impurezas. El modelo desarrollado fue validado comparando previsiones numéricas con datos industriales de evolución de la temperatura superficial en la producción de planchas del acero C-Mn y extendidos a los aceros IF. La medida fue hecha con un termopar aislado de los flujos de agua, en contacto con la superficie de la chapa, durante una carrera de la máquina de colada continua. Fueron extraídas medidas de cada zona de enfriamiento durante 20 carreras de la máquina, estableciéndose entonces una temperatura promedio para cada región.

Como dados de entrada del modelo fueron seleccionados el flujo de agua en cada zona de enfriamiento y la temperatura medida en la plancha. Además, el modelo fue utilizado para investigar las velocidades necesarias de enfriamiento y de fundición para producir planchas de acero IF convencionales. Las figuras 3 y 4 muestran una comparación entre los perfiles de temperatura para la superficie y para el centro de la plancha. Estos perfiles muestran un buen ajuste del modelo con los datos industriales, indicando una colada suave, sin roturas en la plancha. La velocidad de colada impuesta al modelo fue exactamente la misma utilizada en la industria, o sea, $0,81 \mathrm{~m} / \mathrm{s}$.

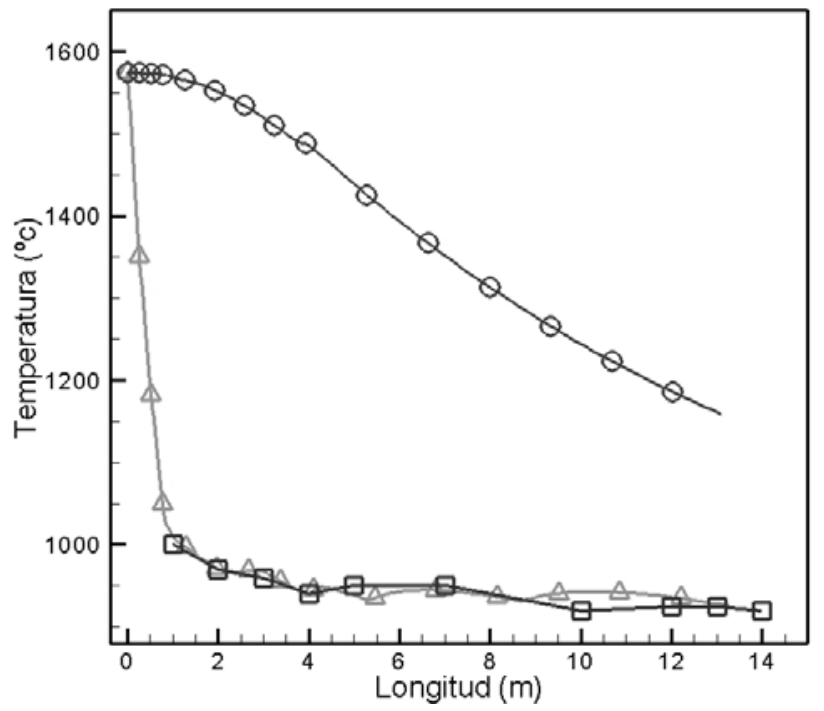

Fig. 3: Colada continua del acero C-Mn $\square$ Simulación de la temperatura superficial $\triangle$ Simulación de la temperatura en el centro $\theta$ Datos industriales

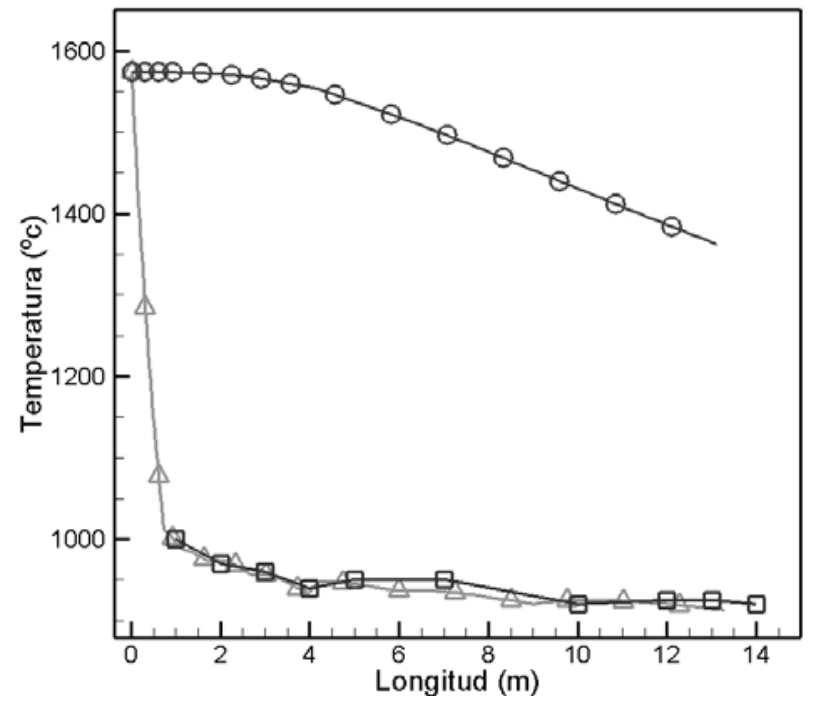

Fig. 4: Colada continua del acero IF $\square$ Simulación de la temperatura superficial $\triangle$ Simulación de la temperatura en el centro $\ominus$ Datos industriales 
Como puede ser observado, la temperatura de la capa pudo mantenerse casi constante para todas las condiciones de fundición simuladas, mientras que la temperatura central se modifica fuertemente. En la figura 2, sin embargo, la temperatura en el centro fue menos afectada debido a que esta clase de aceros presenta baja conductividad térmica y bajo calor específico.

Un coeficiente de transferencia de calor específico es impuesto en cada región para el enfriamiento de la chapa de acero en la superficie superior e inferior de la chapa, como muestra la Fig. 5.

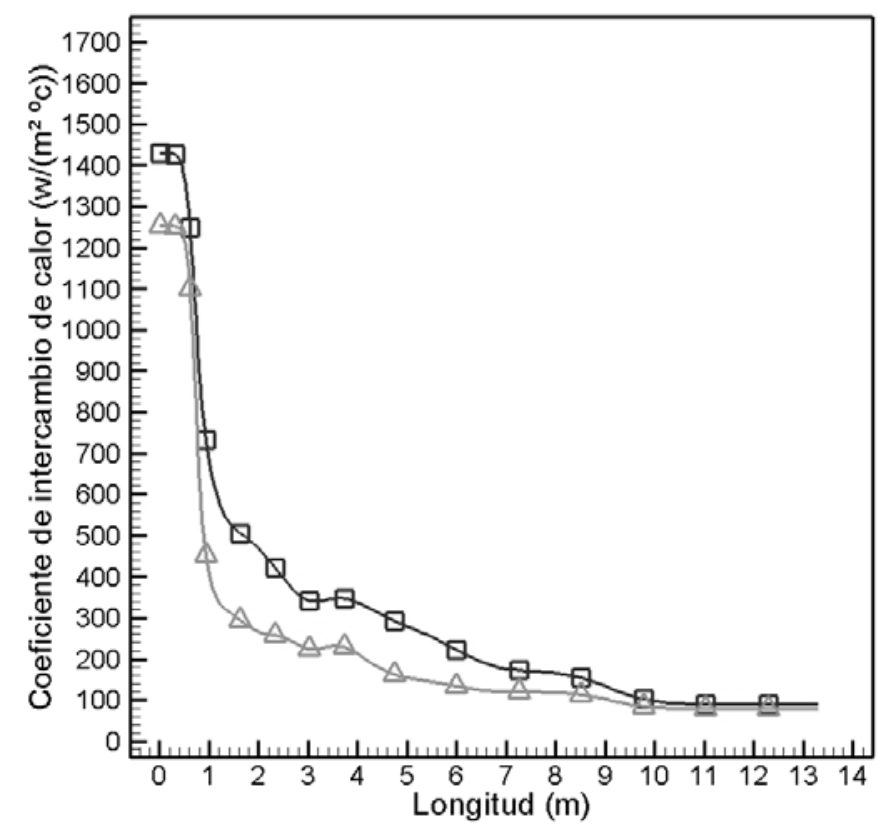

Fig. 5: Coeficiente de intercambio de calor de la superficie inferior y superior. C-Mn $\square ;$ IF $\triangle$ Los campos de distribución de temperatura para los dos casos encima aparecen en las figuras 6 y 7 .

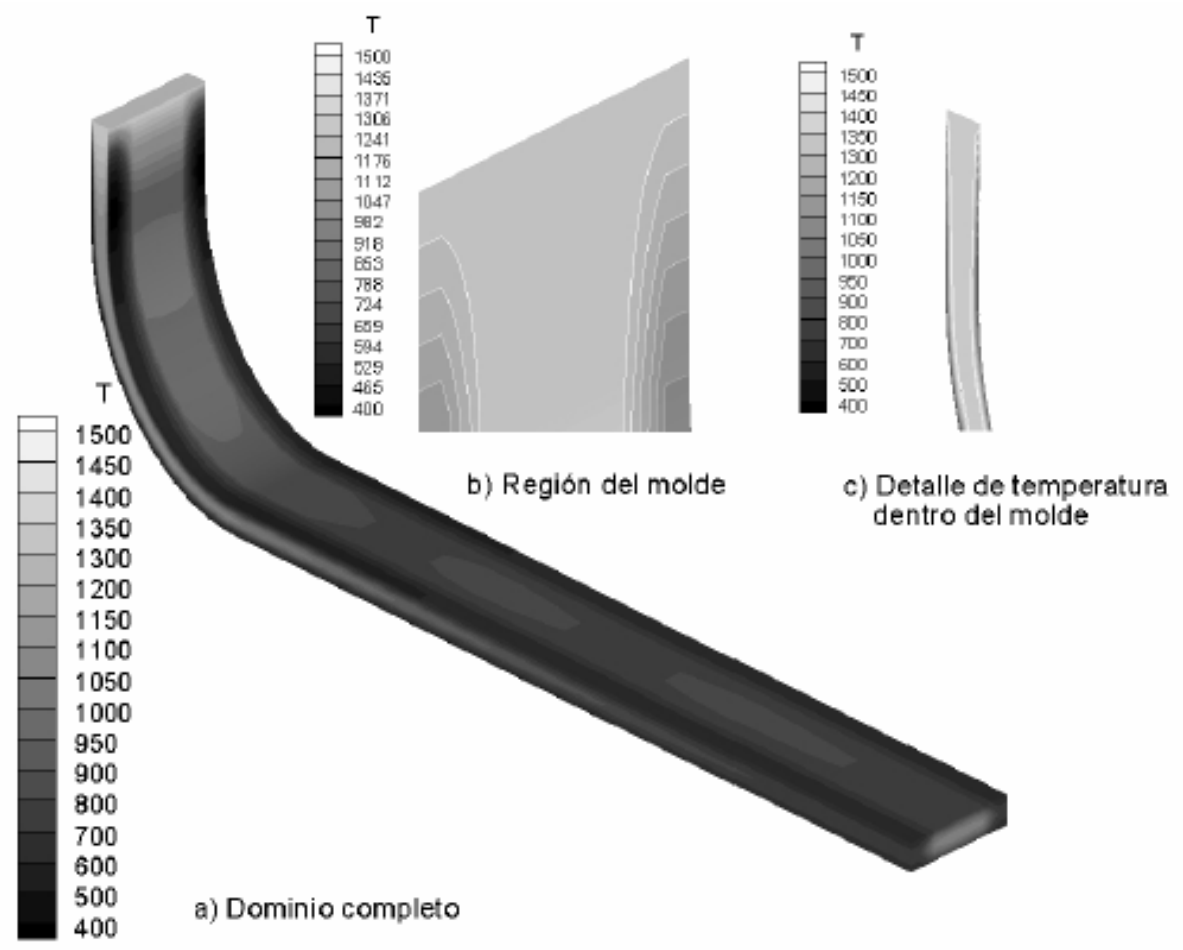

Fig. 6: Perfil de temperaturas para el proceso convencional de colada continua de aceros C-Mn. 


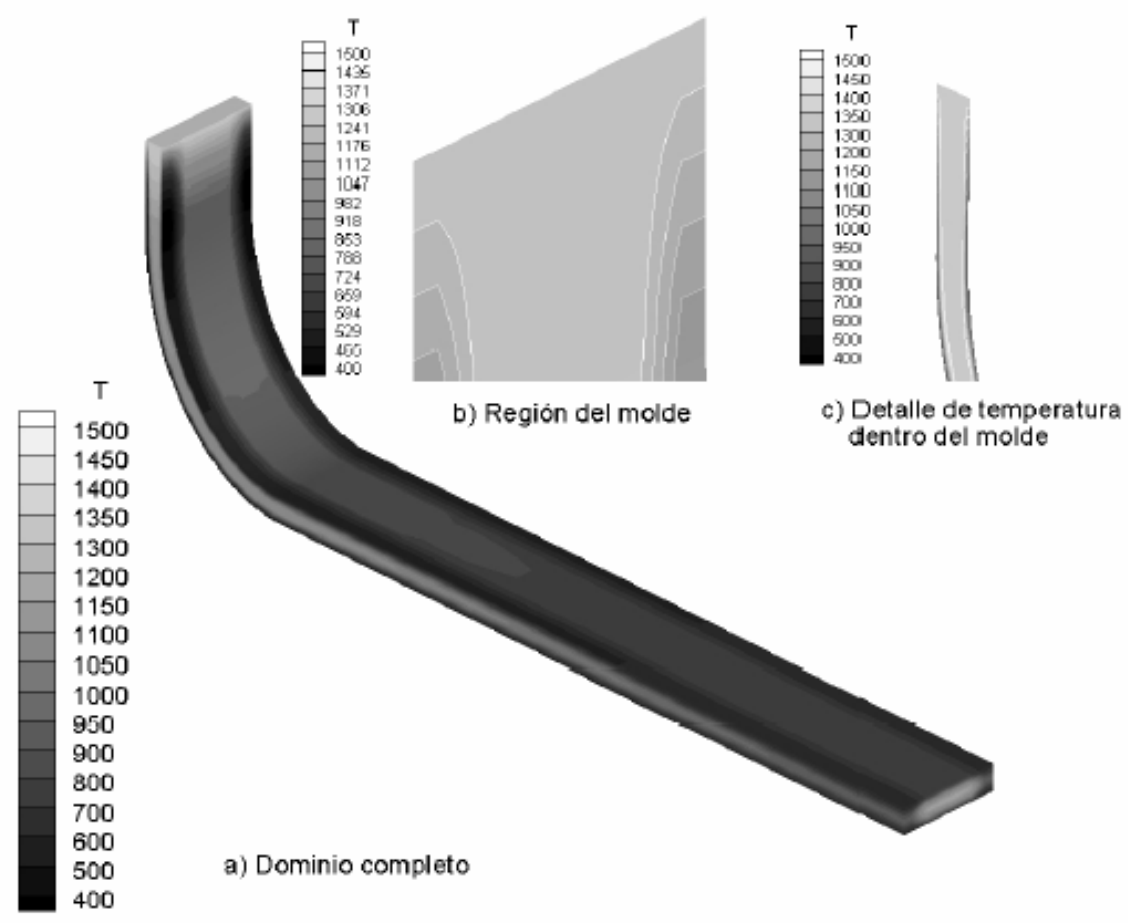

Fig. 7: Perfil de temperaturas para el proceso convencional de colada continua de aceros IF.

La capa solidificada de las chapas fue medida con la intención de investigar la probabilidad de que las mismas se rompan cuando pasen por la región de doblamiento de la máquina de colada continua, como puede ser visto en las Figs. 8, 9 y 10. Como observado anteriormente, la solidificación ocurre más rápidamente en el acero C-Mn mientras que en el acero IF es demorada. A pesar de que este resultado sea considerado crítico, observase que el espesor todavía es seguro en relación a la rotura. El comportamiento del acero IF permite trabajar con velocidades de solidificación superiores lo que aumenta la productividad, haciendo con que el acero IF presente mayor flexibilidad operacional en comparación con el acero C-Mn.

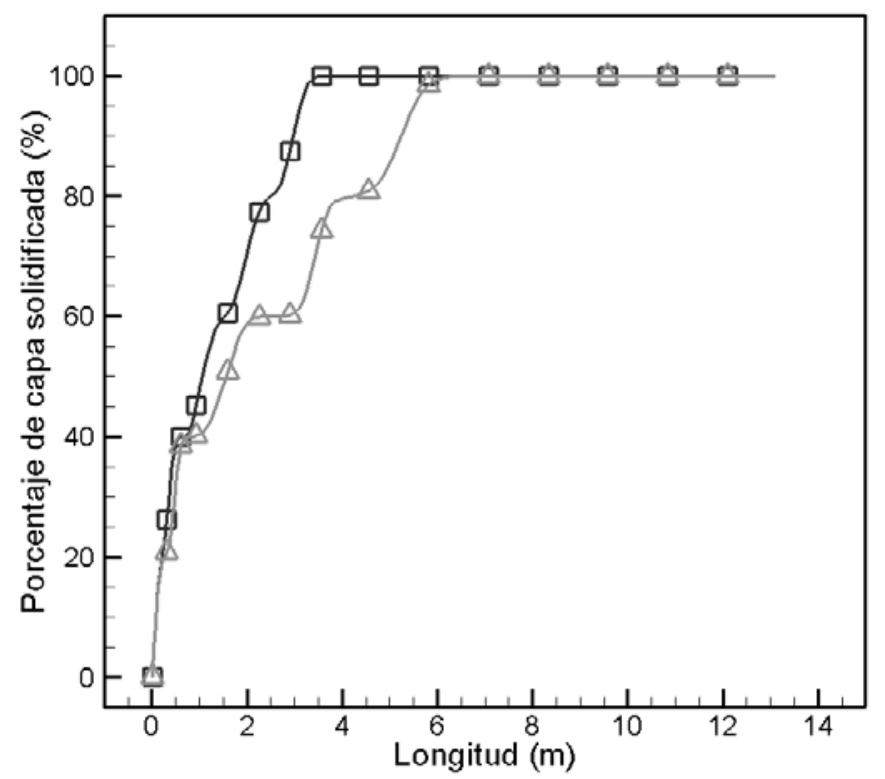

Fig. 8: Porcentaje de capa solidificada. C-Mn $\square ; I_{\triangle}$ 


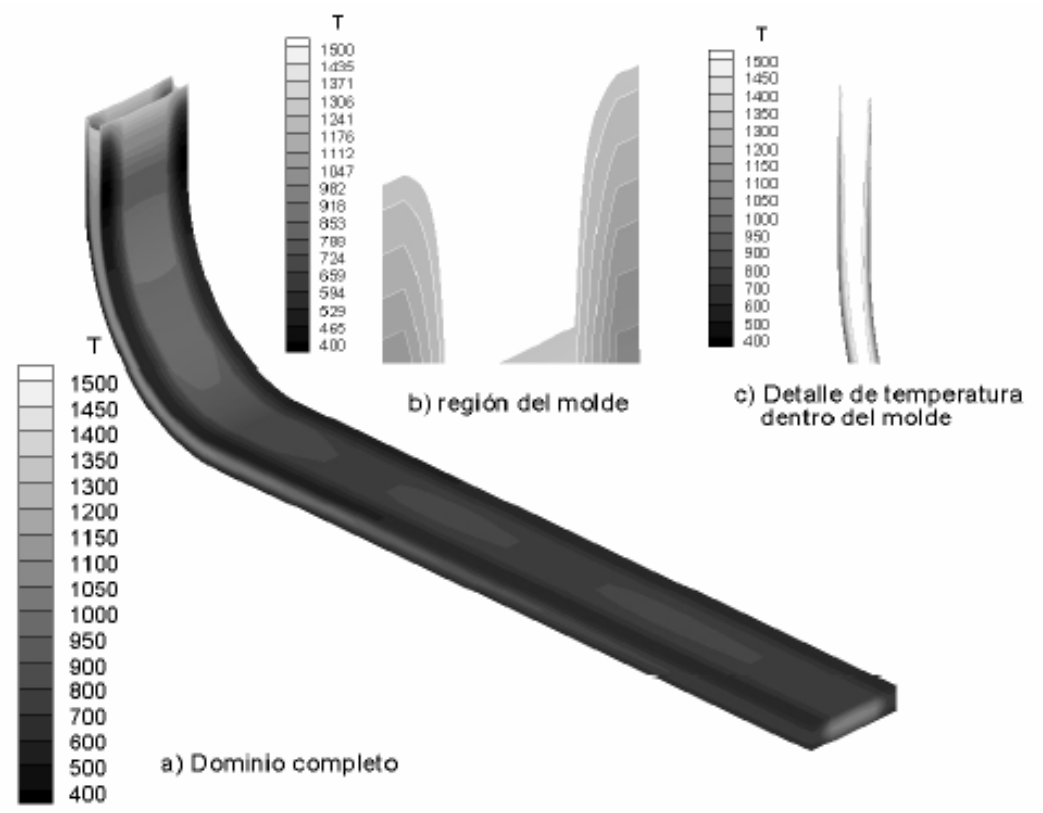

Fig. 9: Perfil de temperatura observando el espesor de la capa solidificada para el acero C-Mn

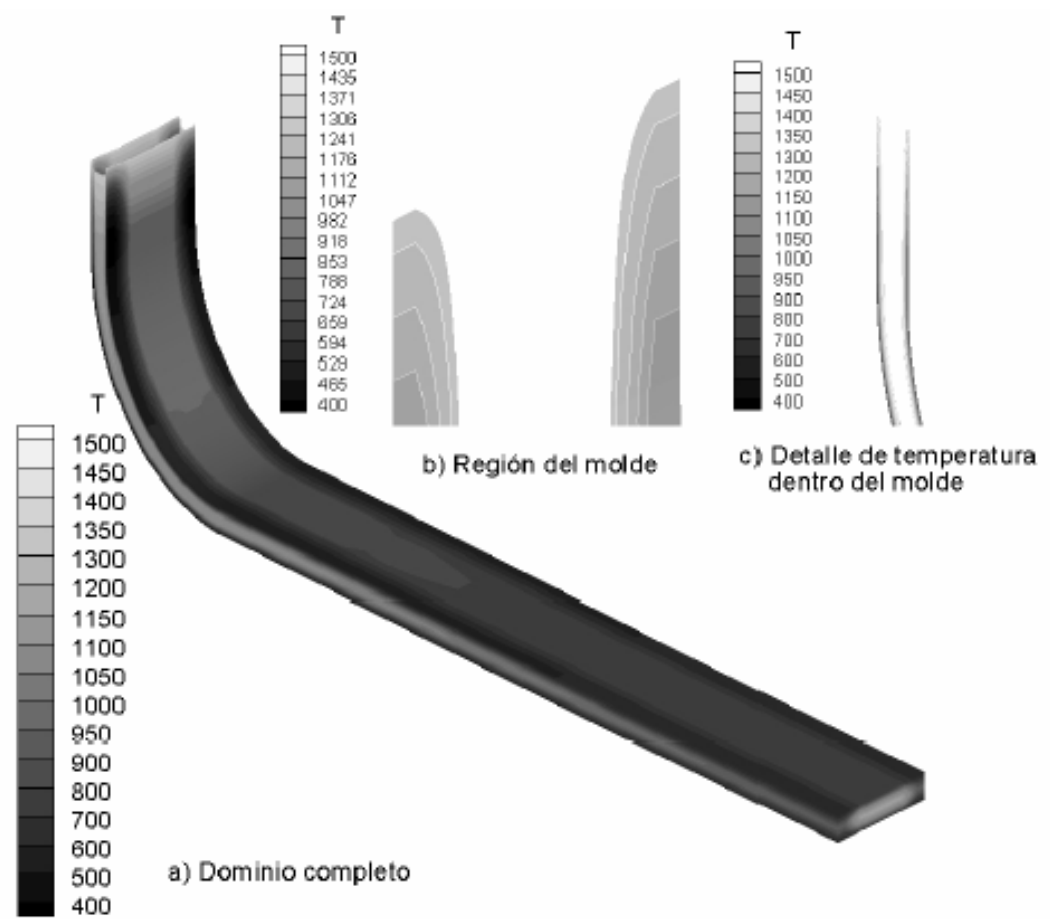

Fig. 10 Perfil de temperatura observando el espesor de la capa solidificada para el acero IF.

\section{CONCLUSIONES}

Este trabajo presentó el desarrollo de un código computacional para simular el proceso de colada continua de aceros IF. El modelo es capaz de simular las condiciones de colada y enfriamiento y se ha aplicado en el modelamiento de un proceso convencional de colada continua de chapas de acero IF. Para validar el modelo se han comparado las previsiones numéricas con datos industriales de temperatura superficial. Los resultados del modelo indican que es posible producir planchas de acero IF convencionales en condiciones de fundición similares a las de planchas de acero C-Mn. Mediante el análisis de la temperatura superficial es posible investigar semejanzas entre los casos simulados que hipotéticamente permitirían condiciones de fundición suave para la colada continua de planchas de acero IF. No obstante, el modelo no es capaz de garantizar las propiedades mecánicas y 
estructurales deseadas en las chapas de acero IF, como fue demostrado previamente en el proceso de colada continua utilizado para validar el modelo mediante comparación con datos industriales.

\section{AGRADECIMIENTOS}

Los autores agradecen a la Fundación de Amparo a la Investigación del estado de Río de Janeiro (FAPERJ) y al Consejo Nacional de Investigación (CNPq) por la ayuda financiera.

\section{REFERENCIAS}

Choudhary, S. K., D. Mazumdar, y A., Ghosh, Mathematical modeling of heat transfer phenomena in continuous casting of steel. ISIJ International: 33(7), 764-774, (1993).

Colin, J. S., y Brandes, E. A., Metals reference book, 5 edición, Butterworths, Londres, Inglaterra, (1976).

Fic, A., Heat transfer analysis of the continuous casting process by the front tracking BEM, Engineering Analysis with Boundary Elements: 24(3), 215-223, (2000).

Ha, M. Y., H. G. Lee, y S. H. Seong, Numerical simulation of three-dimensional flow heat transfer and solidification of steel in continuous casting mold with electromagnetic brake, Journal of Materials Processing Technology: 133, 322-339, (2003).

Holman, J.P., Heat transfer. 5 edición, Mc Graw - Hill Book Company, New york, USA, (1981).

Huespe, A. E, A. Cardona y V. Fachinotti, Thermomechanical model of continuous casting process. Computer Methods in Applied Mechanics Engineering, vol. 182, n. 3-4, p. 439-455, (2000).

Janik, M., H. Dyja, S. Berski y Banaszek, G., Two-dimensional thermomechanical analysis of continuous casting process, Journal of Materials Processing Technology,153-154, 578-582, (2004).

Lan, X. K. y J. M. Khodadadi, Heat transfer and solidification in mold $f$ continuous caster during ladle change, International Journal of Heat and Mass Transfer, 44(5), 953-965, (2001).

Melaaen, M. C., Calculation of Fluid Flows with Staggered and Nonstaggered Curvilinear Nonorthogonal Grids-The Theory, Numerical Heat Transfer-Part B, 21(1), 1-19, (1992).

Moreira, L. P. y J. A. Castro, Modeling the hot rolling process using a finite volume approach. Transactions on Engineering Sciences, 59, 419-430, (2008).

Patankar, V.S., Numerical Heat and Fluid Flor, Hemisphere Publishing Corp., New York, USA (1980).

Peng, X., J. Zhou y Y. Qin, Improvement of temperature distribution in continuous casting moulds through the rearrangement of the cooling water slots, Journal of Materials Processing Technology, 167(3), 508-514, (2005).

Silva, S. P. S., Simulação matemática da influência da composição de carbono do aço na previsão de defeitos no lingotamento contínuo, Tesis de Magister, Universidade Federal Fluminense, Volta Redonda, Brasil. (1996).

Shi, Z. y Z. X. Guo, Numerical heat transfer modeling for wire casting, Materials Science and Engineering A, 365 (1-2), 311-317, (2004).

Thomas, B. G., L. J. Mika, , y F. M. Najjar, , Simulation of fluid flow inside a continuous slab-casting machine. Metallurgical Transactions B: 21B(2), 387-400, (1990).

Zienkiewicz, O. C., Flow of Solids During Forming and Extrusion: Some Aspects of Numerical Solutions, International Journal of Solids and Structures, 14(1), 15-38, (1978). 